\title{
Technical Notes on Mobile Measuring System for Road Passport
}

\section{MOBILE MEASURING SYSTEM TECHNOLOGY}

CDV has developed a new road passport technology. The process included design, processing and development of both technical and software means - the result is a mobile road and traffic data measuring system. The greatest benefit is the complex creation of a road passport using a video recorder. The main observed data are as follows:

- horizontal and vertical road alignment (with the use of GPS)

- video capture of the road at a given density according to road stationing (density is chosen by client or author of passport)

- location of traffic signing, marking and devices (amount of recorded data is chosen by client or author of passport)

- connection to nodal localization system of Road Data Bank Ostrava (managed by the Road and Motorway Directorate of the Czech Republic)

- $\quad$ road video recording

With the CDV's above-mentioned technology, the road passport can be taken on-the-run at normal driving speeds (this principle is called "floating vehicle"), using a vehicle equipped with the described measuring system. This technology is protected by a Czech utility patent (Industrial Property Office \#18277).

\section{ROAD PASSPORT}

The road passport is a complex digital documentation of a road section taken on-the-run in real traffic conditions with a so called "floating vehicle". During documentation, video recorders and other measuring devices are used. All the data are collected by the main unit and is related to time and/or GPS position. After processing, the user can view a video record with custom localization and technical route parameters. The final passport can be used to document road alignment, width parameters and cross-section layout, traffic signing and marking condition, etc. Technical layout is displayed in Fig 1. It includes the main unit ( $\breve{R} J)$, recording module (MU), video device (VCR), portable PC with SW for calibration, measuring and processing (NB), wireless data transfer module and GPS for precise position and time data. The road passport procedure will be described in five following parts.

\subsection{Choosing the route for the road passport}

The selection of route depends on choosing the origin and terminal point of the route in ArcGIS map interface. SW operates with the nodal localization system of the Road Data Bank Ostrava (managed by the Czech Road and Motorway Directorate). The selected route nodes are exported to custom-made Fcar2 SW. The route is related to road network nodes which guarantees data precision..

\subsection{Road passport data collection}

In the course of the route, "events" are recorded. The term "events" includes, for example, traffic sign posts, change of road marking, location of traffic equipment and devices, kilometre posts, etc. 


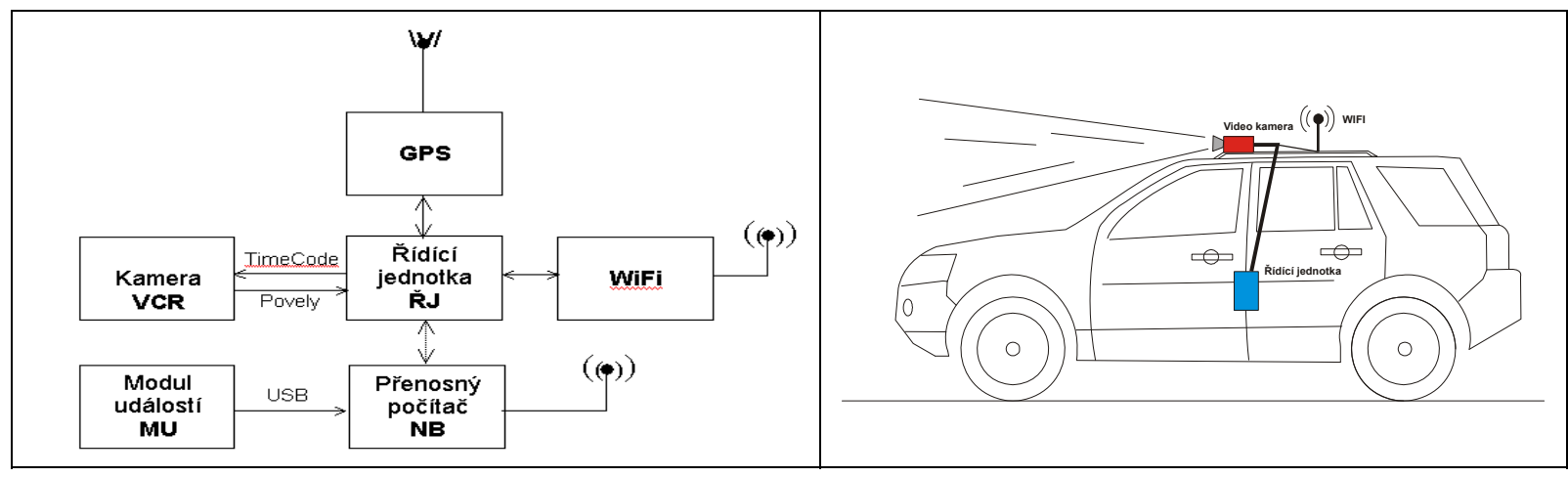

Figure 1: Technical layout of system

Figure 2: Design of measuring vehicle with mobile measuring system

\subsection{Video passport processing}

At this stage, the "raw" passport is being processed, ie. field data are being precised and completed with other parameters. Eventually, the operator can edit the position of events or add new "events" according to viewing the video record in the office. A graphic symbol of a traffic sign is added to each traffic sign "event". The output file is synchronized with the road stationing.

\subsection{Road passport printouts}

Road passport printouts are intended to ease and streamline outputs of the whole passport process. Their objectives are to automate final output documentation in paper form. FCar2 SW disposes of the following printout features: eg. creation of directional signs with destination descriptions or typical bearing constructions (post, gate, cantilever, etc.). Traffic signs positions, precised with the help of a video record, can be supplemented with photographs (video screen captures). Another feature is the export of passport data in the HTML/XML format.

\subsection{Road passport export (FCar2 to ArcGIS)}

Road passports can be processed, visualized and/or edited in the ArcGIS interface. Due to the size of the resulting passport file, it has to be split into parts divided by road network nodes. After the split and export, output files can be viewed in GIS or on the client's PC using the FCar2 data viewer.

\section{CONCLUSION}

The mobile measuring system produces complex digital documentation of the road. Main outputs include a video document of the road, traffic signs localization and a crossfall/gradient analysis. Such a passport can be used in the course of traffic signing/marking implementation, high risk sites investigation, as a background for accident analysis and/or road safety inspection. All resulting data are related to nodal localization system and can be visualized in GIS.

Authors:

J. Ambros \& M. Dont \& R. Striegler*, Transport Research Centre, Czech Republic *Corresponding author: radim.striegler@cdv.cz 